\title{
Revisiting quantum optics with single plasmons
}

Benjamin Vest, Ilan Shlesinger, Marie-Christine Dheur, Eloïse Devaux, Jean-Paul Hugonin, et al.

Benjamin Vest, llan Shlesinger, Marie-Christine Dheur, Eloïse Devaux, Jean-Paul Hugonin, Jean-Jacques Greffet, Gaétan Messin, François Marquier, "Revisiting quantum optics with single plasmons," Proc. SPIE 10722, Plasmonics: Design, Materials, Fabrication, Characterization, and Applications XVI, 107220A (19 September 2018); doi: 10.1117/12.2320322

SPIE Event: SPIE Nanoscience + Engineering, 2018, San Diego, California, United States 


\title{
Revisiting quantum optics with single plasmons
}

\author{
Benjamin Vest ${ }^{\mathrm{a}, \mathrm{b}}$, Ilan Shelsinger ${ }^{\mathrm{a}}$, Marie-Christine Dheur ${ }^{\mathrm{a}}$, Eloïse Devaux ${ }^{\mathrm{c}}$, Jean-Paul Hugonin ${ }^{\mathrm{a}}$, \\ Jean-Jacques Greffet ${ }^{\mathrm{a}}$, Gaétan Messin ${ }^{\mathrm{a}}$, François Marquier ${ }^{\mathrm{a}}$, \\ ${ }^{a}$ Laboratoire Charles Fabry, Institut d'Optique Graduate School, CNRS, Université Paris-Saclay, \\ 91127 Palaiseau Cedex, France; \\ ${ }^{\mathrm{b}}$ Thomas J. Watson Laboratories of Applied Physics, California Institute of Technology, Pasadena, \\ California 91125, USA \\ 'Institut de Science et d'Ingénierie Supramoléculaires, CNRS, Université de Strasbourg, F-67000 \\ Strasbourg, France
}

\begin{abstract}
The growing field of quantum plasmonics lies at the intersection between nanophotonics and quantum optics. QUantum plasmonics investigate the quantum properties of single surface plasmons, trying to reproduce fundamental and landmark quantum optics experiment that would benefit from the light-confinement properties of nanophotonic systems, thus paving the way towards the design of basic components dedicated to quantum experiments with sizes inferior to the diffraction limit. Several groups have recently reproduced fundamental quantum optics experiments with single surface plasmons polaritons (SPPs). We have investigated two situations of quantum interference of single SPPs on lossy beamsplitters : a plasmonic version of the Hong-Ou-Mandel experiment, and the observation of plasmonic N00N states interferences. We numerically designed and fabricated several beamsplitters that reveal new quantum interference scenarios, such as the coalescence and the anti-coalescence of SPPs, or quantum non-linear absorption. Our work show that losses can be seen as a new degree of freedom in the design of plasmonic devices.
\end{abstract}

Keywords: Quantum plasmonics, single plasmon, quantum optics, losses, decoherence

\section{INTRODUCTION}

Surface plasmon polaritons (SPPs) are collective oscillations of electrons that propagate along a metal-dielectric interface [1]. Several groups have reproduced fundamental quantum-optics experiments with such surface plasmons instead of photons, both being bosons. Observation of single-plasmon states [2,3], wave-particle duality [4,5], preservation of entanglement of photons in plasmon-assisted transmission [6-8] and more recently, two-plasmon interference have been reported in a large variety of plasmonic circuits [3, 9-12]. The possibility to generate pairs of indistinguishable single plasmons (SPPs) is an important requirement for potential quantum information applications [13-15].

When dealing with indistinguishable particles, the correlations at the output of a beam splitter are associated to the bosonic or fermionic character of the particles [16]. At first glance, the observation of coalescence appears to be a signature of the bosonic nature of SPPs or photons. However, it has been pointed out that anticoalescence can be observed with photons when using an antisymmetric polarization entangled state [17]. This behavior stems from the introduction of internal degrees of freedom in the wave function: the global photonic state remains symmetric but both the polarization state and the spatial state are antisymmetric. Hence, when a beam splitter is illuminated with this state and if the detectors are not sensitive to the polarization, the situation is similar to the fermionic case and output correlations reveal anticoalescence. This property has been used as a method of analysis of Bell states [18]. These ideas have been further used to mimick fermions with bosons [19]. We also note that anticoalescence of photons have been observed in the context of a quantum eraser experiment, which is also based on the interplay between the spatial state and the polarization state [20]. In all these works, it is assumed that the beam splitter is unitary and therefore, the phase difference between the reflection and the transmission factor is $90^{\circ}$.

Plasmonics: Design, Materials, Fabrication, Characterization, and Applications XVI, edited by

Din Ping Tsai, Takuo Tanaka, Proc. of SPIE Vol. 10722, 107220A · @ 2018 SPIE

CCC code: $0277-786 \mathrm{X} / 18 / \$ 18 \cdot$ doi: $10.1117 / 12.2320322$ 
As pointed out in Refs. [21,22], it is possible to change this phase difference when considering losses in the beam splitter. Indeed, it is shown that the presence of scattering or absorption on the beam splitter relaxes constraints on the reflection and transmission factors, allowing the control of their relative phase. The authors of Refs [21,22] predicted in particular novel effects including coherent absorption of single photon and N00N states [23,24]). While losses are detrimental for the observation of squeezed states, they can thus be seen as a degree of freedom in the design of plasmonic devices revealing new quantum-interference scenarios : in this paper, we explore two situations based on the interference of two indistinguishable single plasmons on a lossy plasmonic chip.

\section{PLASMONIC HONG-OU-MANDEL EXPERIMENT}

Here we report the observation of two-plasmon quantum interference between two freely-propagating, non-guided SPPs interfering on lossy plasmonic beam splitters in two sets of experiments. Firstly, we designed several plasmonic beam splitters with different sets of reflection and transmission factors, that are used in a plasmonic version of the Hong-OuMandel (HOM) experiment [25], in which the input state is a symmetric spatial state, and has no internal degrees of freedom: the polarization of the SPPs is fixed. Depending on the plasmonic beam splitters, coincidences measurements lead either to a HOM-like dip, i.e. a signature of plasmon coalescence, or a HOM-peak, that we associate to plasmon anticoalescence. In the latter case, the anticoalescence is fundamentally related to the beam splitter itself, which introduces antisymmetry in the system [26]. This effect reminds that the bosonic nature of particles, here surface plasmons, does not imply bunching at a beam splitter. Secondly, we report the first observation of interferences of plasmonic N00N states freely propagating along a gold-air interface and interfering on a lossy beamsplitter. In this work at the interface between plasmonics and quantum optics, we will study the interplay between quantum interferences, plasmonic confinement and losses.

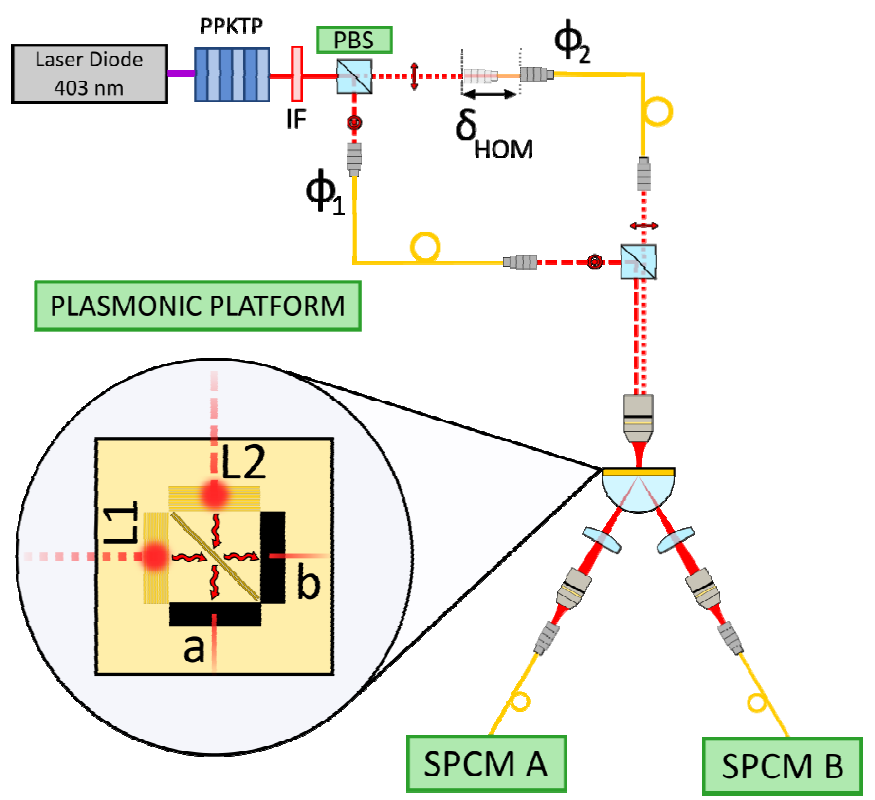

Fig 1. Sketch of the experimental setup. A PPKTP crystal is pumped by a laser diode at $403 \mathrm{~nm}$ and delivers pairs of orthogonally polarized photons at $806 \mathrm{~nm}$. An interference filter (IF) removes the remaining pump photons. The nearinfrared photons are separated by a polarizing beamsplitter (PBS), and injected in monomode fibers. They therefore excite the photonic modes $\Phi 1$ or $\Phi 2$, that are respectively converted by the SPP launchers L1 and L2 into plasmonic modes on a plasmonic platform. One of the fiber collimator input is placed on a translation, so that a delay $\delta \mathrm{HOM}$ between the two SPPs can be settled changing the optical path of one of the photons after the PBS. The two single SPPs are recombined on a plasmonic beamsplitter and finally out-coupled to photonic modes a and $\mathrm{b}$. The light is transmitted form the substrate to the free space by a hemispherical lens before being collected by two $75 \mathrm{~mm}$-focal-length lenses at both output ports of the platform, and is injected by two focusing objectives in multimode fibers. SPCMs A and B record detection counts respectively from output modes $a$ and $b$, and measure coincidences between the detectors. 
Our experimental setup is based on a source of photon pairs (Fig. 1). The photons of a given pair are sent to two photonto-SPP converters, located at the surface of a plasmonic test platform. The photon number statistics are conserved when coupling the photonic modes to a plasmonic mode on such a device [27], so that pairs of incident single-photons are converted into two single SPPs. These SPPs freely propagate on the metallic surface towards the two input arms of a plasmonic beam splitter. After the beam splitter, the SPPs that reach the output of the platform are converted back to photons to be detected by single photon counting modules (SPCMs).

(A)

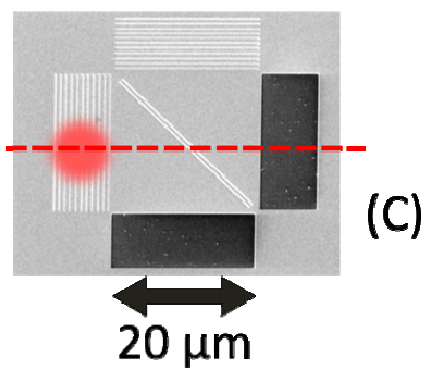

(B)
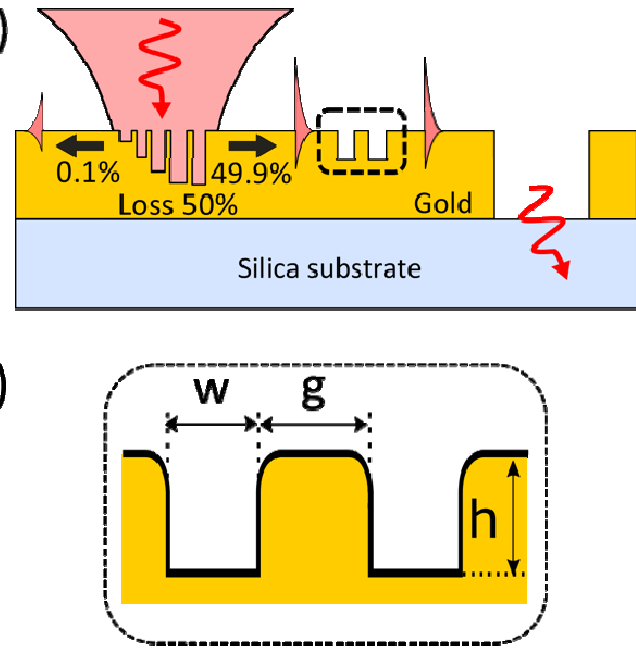

Fig 2. Presentation of the plasmonic platform.(A) SEM picture of a plasmonic platform. The dotted red line represents the direction along which the section is depicted in (B). The red spot represents an incident gaussian beam on the SPP launcher. (B) Sectional drawing of the device. On the left, the first structure is a photon-to-SPP coupler. When single photons reach the grating, single SPPs are launched unidirectionaly toward the plasmonic beamsplitter (SPBS) (grooved doublet). The remaining SPPs propagate to the large outcoupling slits. With an efficiency of about $50 \%$, SPPs are converted back to photons in the silica substrate. (C) Close-up look on the SPBS. Dimensions of the SPBS are defined by three parameters, the grooves width $w$, the metal gap between the grooves $g$ and the height of the groove $h$, that affect the reflection and transmission factors of the beamsplitter.both output ports of the platform, and is injected by two focusing objectives in multimode fibers. SPCMs A and B record detection counts respectively from output modes $\mathrm{a}$ and $\mathrm{b}$, and measure coincidences between the detectors.

The plasmonic platform consists in several elements that are etched on a $300 \mathrm{~nm}$-thick gold film on top of a silica substrate, on a total $40 \times 40 \mu \mathrm{m}$ footprint (see Fig. 2). The input channels of the plasmonic platform are made of two unidirectional launchers (denoted as L1 and L2). Those asymmetric 11-grooves gratings have been designed to efficiently couple a normally incident Gaussian mode into directional SPPs [28]. The SPPs generated by each launcher then freely propagate and recombine on the surface plasmon beam splitter (SPBS). It is made of two identical grooves in the metallic surface (see Fig.2 (c)), oriented at $45^{\circ}$ with respect to the propagation direction of waves launched by L1 and L2. The succession of metal and air allows a scattering process that generates both a transmitted and a reflected SPP [29], but this also introduces losses. The complex reflection and transmission factors $r=|r| e^{i \varphi_{r}}$ and $t=|t| e^{i \varphi_{t}}$ of the SPBS are functions of the geometrical parameters of the SPBS (see Fig.2 (c)). In particular, it is possible to control the phase difference $\varphi_{r t}=\varphi_{r}-\varphi_{t}$. This phase control affects the interferences. The SPPs then propagate towards two large out-coupling strip slits. They are decoupled into photons propagating in the glass substrate on the rear of the platform.

For a lossless balanced beam splitter, energy conservation and unitary transformation of modes at the interface imposes $t= \pm i r$ and $|t|=|r|=\frac{1}{\sqrt{2}}$ so that the phase difference between $r$ and $t$ is $\varphi_{r t}= \pm 90^{\circ}$. When placed at the end of a Mach-Zehnder interferometer, the two outputs of the beam splitter deliver two sinusoidal interference signals that display a phase-shift $2 \varphi_{r t}= \pm 180^{\circ}$.. It follows that a maximum on a channel corresponds to a minimum on the other channel as 
expected from energy conservation arguments. The situation is different in our experiment; a single SPP is transmitted with probability $|t|^{2}$, reflected with a probability $|r|^{2}$, but can be absorbed or scattered with a probability $1-|r|^{2}-$ $|t|^{2}$. For a balanced SPBS, in presence of losses, $r$ and $t$ are constrained by the following inequality [13]:

$$
|t \pm r|^{2} \leq 1
$$

where the equality holds only if there are no losses. The previous relation releases all constraints on $2 \varphi_{r t}$. In other words, losses can here be considered as a new degree of freedom. It is therefore possible to design several beam splitters where the amplitude of $r$ and $t$ and the relative phase $\varphi_{r t}$ can be modified. As a direct consequence, interference fringes from both outputs of the BS can be found experiencing an arbitrary phase shift.

Controlling those properties of the SPBS strongly affects the detection of events by the two SPCMs. It has been shown [21] that the coincidence detection probability, i.e. the probability for one particle pair to have its two particles emerging from separate outputs of the beam splitter can be expressed as:

$$
\quad P\left(1_{a}, 1_{b}\right)=|t|^{4}+|r|^{4}+2 \Re\left(r^{2} t^{2}\right) I(2)
$$

where $a$ and $b$ label the output ports of the beamsplitter, and $I$ is an overlap integral between the two particles wavepackets. For non-overlapping wavepackets, $\mathrm{I}=0$ and the previous relation reduces to:

$$
P_{c l}\left(1_{a}, 1_{b}\right)=|t|^{4}+|r|^{4}(3)
$$

The particles impinging on the SPBS behave like two independent classical particles, as indicated by the subscript $c l$. For an optimal overlap between the particles $(\mathrm{I}=1)$, the coincidence probability can be written:

$$
P_{q u}\left(1_{a}, 1_{b}\right)=\left|t^{2}+r^{2}\right|^{2}=P_{c l}\left(1_{a}, 1_{b}\right)+2 \Re\left(r^{2} t^{2}\right)(4)
$$

where the subscript $q u$ denotes the presence of the quantum interference term $2 \Re\left(r^{2} t^{2}\right) I$.

We now consider two cases. If $t= \pm i r$, the probability $P_{q u}$ reaches zero. This is the same antibunching result that is obtained for a non-lossy beam splitter (15). This is the so-called HOM dip in the correlation function. If we now consider $t= \pm r$ and $|t|=|r|=\frac{1}{2}$ we get $P_{q u}\left(1_{a}, 1_{b}\right)=2 P_{c l}\left(1_{a}, 1_{b}\right)$. Here, we expect a peak in the correlation function.

The plasmonic chips were designed by solving the electrodynamics equations with an in-house code based on the aperiodic Fourier modal method [30]. Numerical simulations allowed us to find the geometrical dimensions of the beam splitter required for the two previous configurations $t= \pm$ ir or $t= \pm r$ with $|t|=|r|=\frac{1}{2}$ respectively, that is $25 \%$ of the incident energy is transmitted, $25 \%$ is reflected and the amount of non-radiative losses on the beamsplitters is $50 \%$. We fabricated two corresponding samples denoted as samples I and II respectively. The features of each beam splitter are reported in Table 1. We characterized the phase difference between $r$ and $t$ by an interferometric method. We used the plasmonic beamsplitter as the output beam splitter of a Mach-Zehnder interferometer. We splitted an $806 \mathrm{~nm}-\mathrm{CW}$ laser beam in this interferometer and recorded the interference fringes at both output ports of the setup when increasing the relative delay $\delta_{\text {Hом }}$. We then measured the average phase difference between the two signals recorded on the two output channels in order to get $\varphi_{r t}$. 


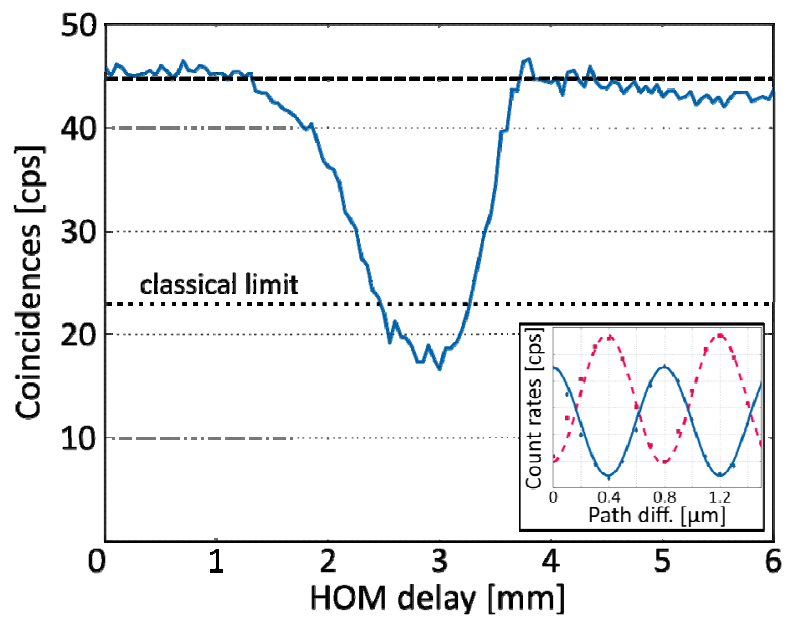

Fig 3. Observation of a plasmonic Hong-Ou-Mandel coalescence effect with freely propagating single SPPs on sample I. The plot displays the coincidence count rates with respect to the HOM delay $\delta \mathrm{HOM}$ between both particles. The delay is indicated as a relative measurement starting from the initial position of the optical apparatus. The coincidences counts have been recorded every $50 \mu \mathrm{m}$, with a five minutes integration time, that is long enough to limit uncertainty on the count rate below 1 coincidence per second. The contrast of the dip is approximately, above the quantum limit at $50 \%$. The inset displays short interferograms of the classical fringes recorded by SPCMs A and B. Similarly to the lossless configuration, the observed sine waves are in phase opposition.

Figure 3 is a plot of the coincidence rate with respect to the HOM delay $\delta_{\text {Hом }}$ between both arms when sample I is used. The inset is a plot of the sinusoidal fringes obtained at the outputs of the beam splitter when illuminating with a laser at $806 \mathrm{~nm}$. It is seen that the fringes are in phase opposition, confirming the $90^{\circ}$ phase-shift between $r$ and $t$. The plot displays an HOM-like dip, with a $61 \%$ contrast, unambiguously in the quantum regime beyond the $50 \%$ limit [32]. This result is analog to the coalescence effect observed in two-photon quantum interference and confirms the bosonic behavior of single plasmon, here achieved for the first time with freely-propagating, non-guided SPPs on a gold surface.

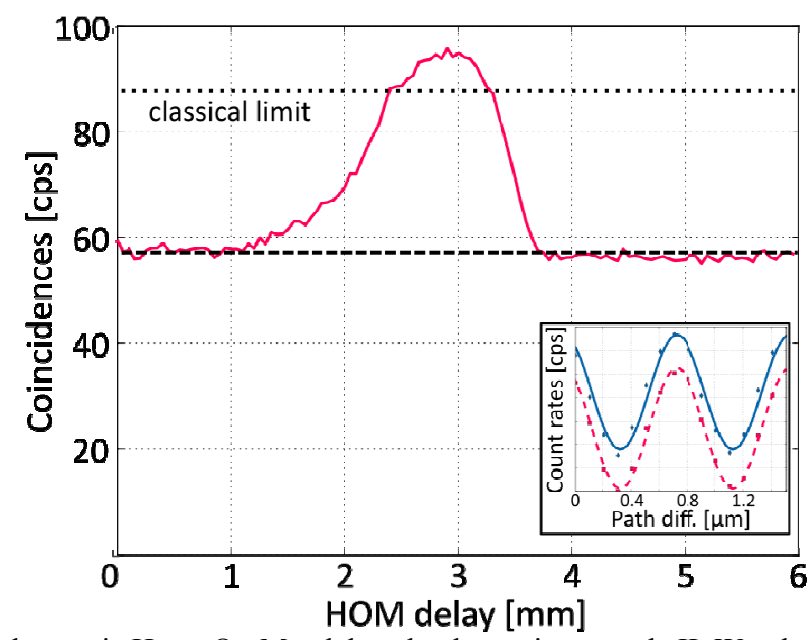

Fig 4. Observation of a plasmonic Hong-Ou-Mandel peak when using sample II. We plotted the coincidence count rate between SPCMs A and B for a varying delay between the particles interfering on the SPBS, and under the same experimental conditions as previously. The contrast of the peak is .Here, the SPBS coefficients have been chosen so that the classical sine fringes are in-phase (see inset). This observation can be interpreted as an anti-coalescence effect. 
Table 1. Dimensions of the plasmonic platform samples under study, as measured by a scanning electron microscope (width w and metal gap g) and atomic force microscope (groove depth h). Notations refer to Figure 2(c). The fourth line reports expected values for the reflection and transmission factors $r$ and $t$ based on the numerical simulations of the target design. The last line reports estimations of the relative phase between the reflexion and transmission coefficients after characterization. Numbers between parenthesis are the target dimensions and relative phase of the devices as designed by numerical simulations.

\begin{tabular}{|c|c|c|}
\hline & Sample I & Sample II \\
\hline $\mathrm{w}[\mathrm{nm}]$ & $171(180)$ & $289(320)$ \\
\hline $\mathrm{g}[\mathrm{nm}]$ & $145(140)$ & $250(280)$ \\
\hline $\mathrm{h}[\mathrm{nm}]$ & $140(120)$ & $150(140)$ \\
\hline$|\mathrm{r} / /| \mathrm{t} \mid$ & $(0.42 / 0.42)$ & $(0.5 / 0.48)$ \\
\hline $2 \varphi_{r t}$ & $170^{\circ}\left(180^{\circ}\right)$ & $10^{\circ}\left(0^{\circ}\right)$ \\
\hline
\end{tabular}

We then move to the next sample, beam splitter II. Inset of Fig. 4 shows that classical fringes at the output are in-phase. In this case, orthogonality is not preserved between output modes of the SPBS. Two-particle quantum interference experiment is now characterized by an HOM-peak, an increase in coincidence rate with respect to the classical case. The contrast is around $70 \%$, once again in a clear quantum regime. The peak illustrates that when combining on this beam splitter, SPPs tends to emerge from two different outputs. This anti-coalescence effect highlights the fundamental role of the SPBS in quantum interference.

\section{PLASMONIC NO0N STATES INTERFERENCES}

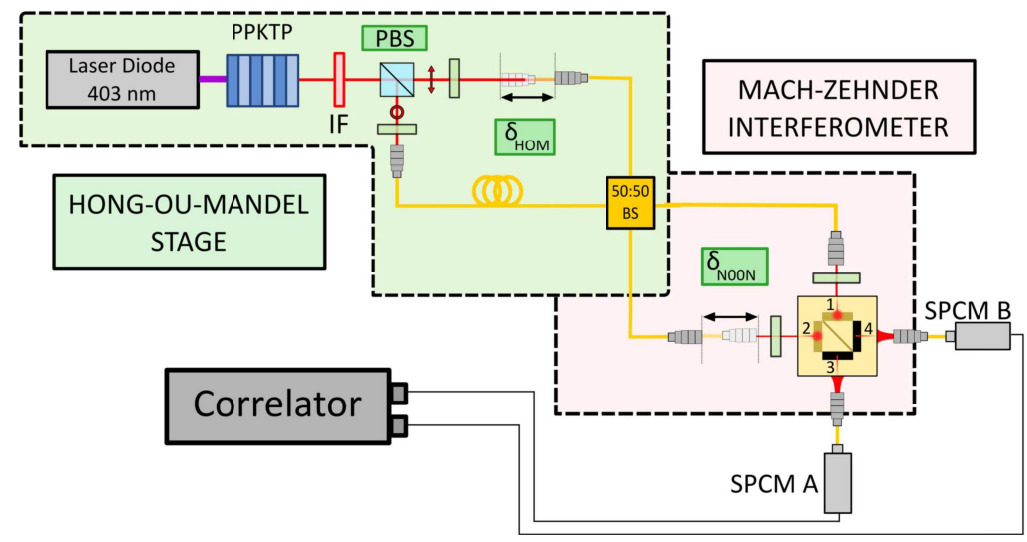

Fig 5. Sketch of the N00N setup. A PPKTP crystal is pumped by a laser diode at $403 \mathrm{~nm}$ and delivers pairs of orthogonally polarized photons at $806 \mathrm{~nm}$. An interference filter (IF) removes the remaining pump photons. A first stage provides the N00N state by two photon interference in a fiber beamsplitter. The outputs of the HOM stage are connected to a Mach-Zehnder interferometer. The near-infrared photons are separated by a polarizing beamsplitter (PBS), and excite the photonic modes, that are, respectively, converted by the SPP launchers 1 and 2 into plasmonic modes on a plasmonic platform. A delay $\delta \mathrm{N} 00 \mathrm{~N}$ between the two arms can be settled changing the optical path of one of the photons after the PBS. The two paths are recombined on a plasmonic beamsplitter and finally outcoupled to photonic modes. SPCMs A and B record detection counts, respectively, from output modes a and b, and measure coincidences between the detectors. 
We now move to a second set of experiments dedicated to the study of plasmonic N00N states. These N00N states are of the form $\langle\psi\rangle=\frac{|\mathrm{N}, 0\rangle+|0, \mathrm{~N}\rangle}{\sqrt{2}}$, i.e. a superposition state where $\mathrm{N}$ particles are in one arm and none in the other arm of a two branch interferometer. N00N states are particularly interesting when performing quantum interferences since they offer the possibility to reduce phase measurement uncertainties below the shot noise limit by a factor $\frac{1}{\sqrt{N}}$ [33]. Here we report the first observation of interferences of plasmonic N00N states freely propagating along a gold-air interface and interfering on a lossy beamsplitter. In this work at the interface between plasmonics and quantum optics, we will study the interplay between quantum interferences, plasmonic confinement and losses. As opposed to quantum optics experiments in vacuum, losses are expected to play a key role in the plasmonic interferences for two reasons. First, propagation losses will be revisited for plasmonic N00N states. Second, we use a lossy beamsplitter which enables us to modify the phase difference between reflection and transmission coefficients. It has been shown that this effect may induce nonlinear absorption [16].

Upstream of the first stage, we generate pairs of orthogonally polarized photons at $\lambda=806 \mathrm{~nm}$ thanks to a single photon down-conversion process in a periodically poled potassium titanyl phosphate (PPKTP) crystal pumped by a laser diode at $403 \mathrm{~nm}$. The photons of a single pair are separated and their polarizations are aligned along the same direction before being injected in the two inputs of a fibered beamsplitter (FBS). One of the input of this FBS is mounted on a translation stage that allows us to control the relative delay $\delta \mathrm{HOM}$ between both particles, such that this entire first part of the setup reproduces a standard photonic Hong-Ou-Mandel (HOM) experiment stage. When the position $\delta \mathrm{HOM}$ is chosen so that the delay between photons is set to zero, the two particles experience coalescence and the output two-particle state is now a N00N state:

$$
\lfloor\psi\rangle=\frac{\left|2_{1}, 0_{2}\right\rangle+\left|0_{1}, 2_{2}\right\rangle}{\sqrt{2}}
$$

where the subscripts 1 and 2 refer to the outputs of the beamsplitter. The plasmonic N00N state interferences are observed thanks to a second hybrid MZ interferometer that introduces a second delay $\delta_{\mathrm{N} 00 \mathrm{~N}}$. The outputs of the previous photonic HOM stage are now connected to the arms of the interferometer, each one being associated to an input of the previously mentionned plasmonic platform, where the quantum interference between plasmon states takes place.

The coincidence detection probability can be computed from the expression of the N00N state and the beamsplitter relations linking the input modes 1 and 2 to the output modes 3 and 4 . First we write the annihilation operators related to the beamsplitter modes.

$$
\begin{aligned}
& a_{3}=t a_{1}+r a_{2} e^{i \varphi} \\
& a_{4}=r a_{1}+t a_{2} e^{i \varphi}
\end{aligned}
$$

where

$$
\varphi=\frac{2 \pi \delta_{N 00 N}}{\lambda}
$$

is the relative phase delay introduced between the arms of the N00N interferometer. The coincidence detection probability can be expressed as:

$$
P\left(1_{3}, 1_{4}\right)=\left\langle\psi\left|\widehat{N_{3} N_{3}}\right| \psi\right\rangle
$$

where $\widehat{N_{3}}=a_{3}^{\dagger} a_{3}$ and $\widehat{N_{4}}=a_{4}^{\dagger} a_{4}$ are the number operators of channels 3 and 4 . By using the previous relations, we get: 


$$
P\left(1_{3}, 1_{4}\right)=2|t|^{2}|r|^{2}(1+\cos (2 \varphi))
$$

The coincidence detection probability thus oscillates twice as fast as the dephasing introduced by the path difference between the two arms. This is due to the fact that the propagation phase accumulation for a Fock state with $\mathrm{N}$ particles, $|N\rangle$ is $\mathrm{N}$ times higher than for a single particle state. Hence we expect to observe interference fringes when recording coincidence count rates for various delays $\delta_{\mathrm{N} 00 \mathrm{~N}}$ that oscillate with half the wavelength of the incident light $\frac{\lambda}{2}$. This is the so-called phase super-resolution that is sought when dealing with $\mathrm{N} 00 \mathrm{~N}$ state interferometry. It is also relevant to point out that, as opposed to the single particle interference case, the N00N state interferences coincidences do not depend on the phase relation between the beamsplitter's reflection and transmission factors $r$ and $t$. Nevertheless it will have an influence on the number of particles that are absorbed as it will be discussed in the last section.

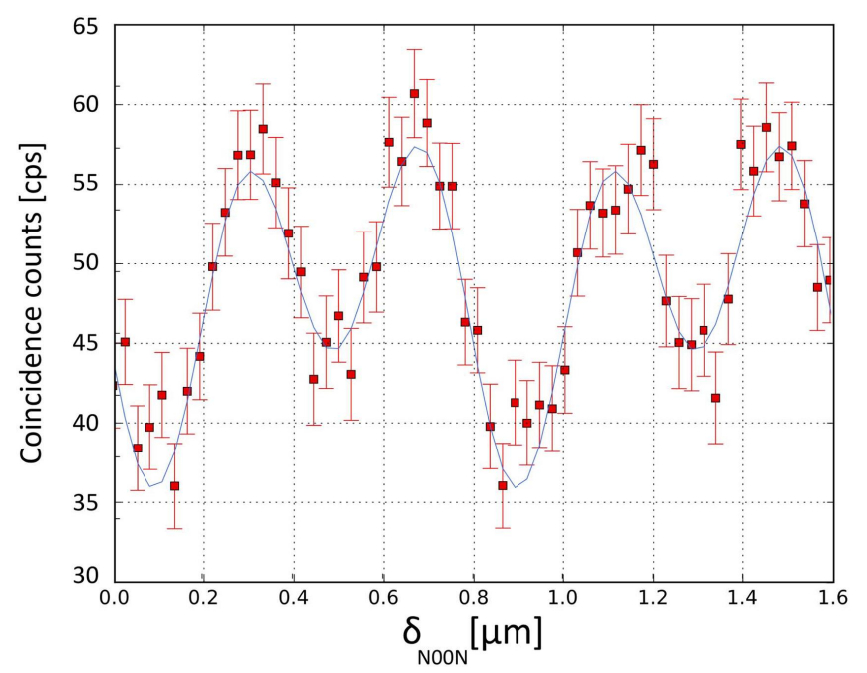

Fig 6. Raw data of the coincidence counts of both detectors as a function of the MZ interferometer path difference $\delta \mathrm{N} 00 \mathrm{~N}$. The solid line is a sum of two sinusoids at $\lambda=806 \mathrm{~nm}$ and $\lambda / 2$ with fitted phases.

Fig. 6 is a plot of the raw coincidences count rate when illuminating the interferometer with $\mathrm{N} 00 \mathrm{~N}$ states $(\mathrm{N}=2)$ for increasing delay $\delta_{\mathrm{N} 00 \mathrm{~N}}$. The signal has been integrated over $10 \mathrm{~s}$. The data show fringes with a period close to $400 \mathrm{~nm}$ which seem to be modulated by a signal of larger wavelength. The fast Fourier transform (FFT) spectrum displayed on Fig. 7 indeed shows that the coincidence signal is the sum of two contributions: one main contribution at a frequency $\frac{2}{\lambda}$ and a smaller one at $\frac{1}{\lambda}$, that slightly distorts the main oscillations observed, with $\lambda=806 \mathrm{~nm}$ the wavelength of the incoming photons. A sum of two sinusoids at $\lambda=806 \mathrm{~nm}$ and $\lambda / 2$ with the corresponding amplitudes given by the FFT of the data and with the phase as a free parameter have been plotted in Fig. 6 . The two oscillations can then be understood as the expected $\mathrm{N} 00 \mathrm{~N}$ interference resulting in coincidences oscillating at twice the frequency of the incoming light which are modulated by a residual signal at the frequency of the photons. This residual perturbation of the coincidence fringes is related to a single-particle interference onto the SPBS. It can indeed be related to the initial depth of the unperfect HOM dip, that characterizes the presence in the setup of unbunched pairs of particles. We show on Fig. 8 the coincidence signal once the contribution at $\lambda$ has been numerically removed by applying a band-stop filter to the FFT around the SP frequency ( between $0.65 \lambda$ and $1.3 \lambda$ ). This provides a clean picture of the signal, that we can fit in order to precisely estimate the frequency of the oscillation, with respect to the initial calibration that has been carried out on the single-plasmon interferences signal. We finally get a period of $397 \pm 10 \mathrm{~nm}$, which is in good agreement with the expected value around $403 \mathrm{~nm}$. The observed signal exhibits a contrast around $20 \%$, obviously much less than the ideal case of a unity visibility of the N00N fringes that can be theoretically reached. We attribute this degradation to many sources in our experimental setup: a non-perfect balancing of the SPBS and more generally of both interferometers involved in the experiment, non-optimal HOM generation of the biphoton state and the limited overlap of the modes propagating freely on the gold surface. 


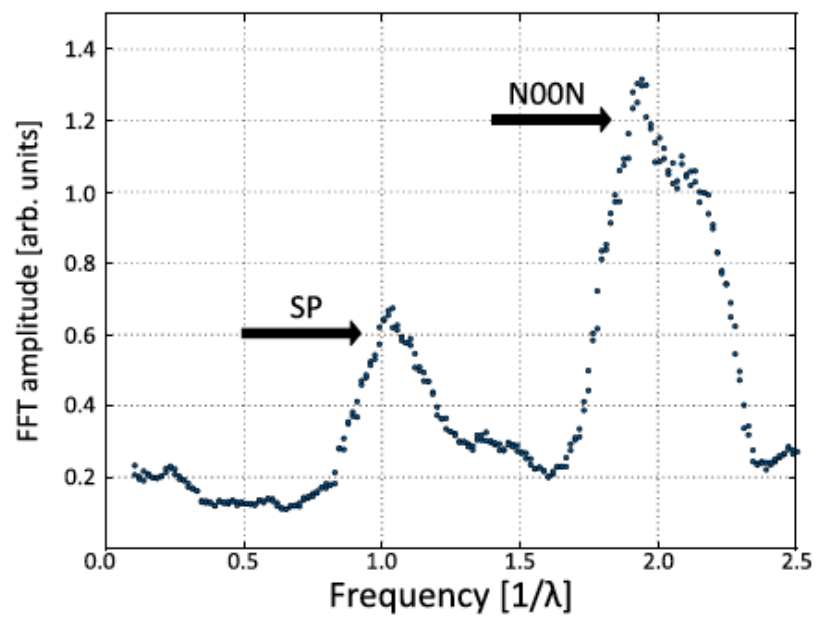

Fig 7. Fast Fourier transform of the raw coincidence counts of figure 6 . The spectrum is plotted as a function of the inverse of the down-converted signal wavelength $\lambda=806 \mathrm{~nm}$. The two arrows are a guide to the eye indicating the contribution of the single plasmon (SP) states with a wavenumber $1 / \lambda$ and of the N00N states at twice that wavenumber.

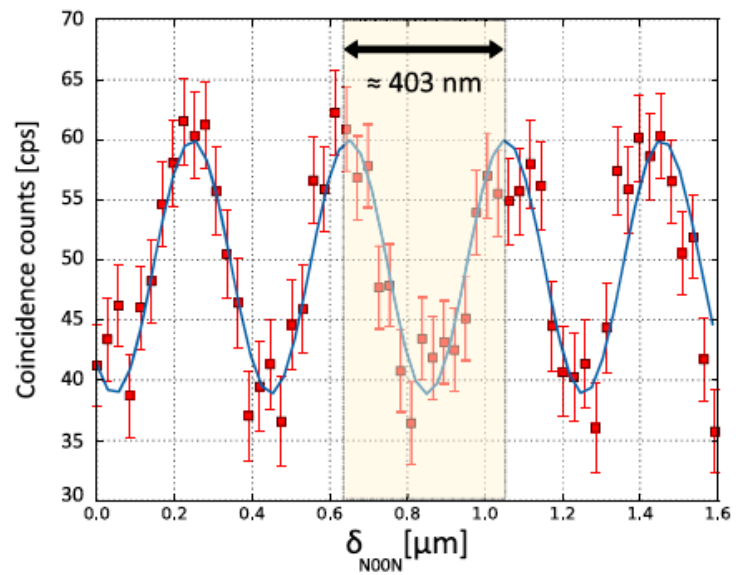

Fig. 8. Coincidence counts (squares) with error bars as a function of theMZinterferometer path difference $\delta$ Noon when the contribution of SP states has been numerically filtered. The solid line is a sinusoid at twice the frequency than the incident light

Two different sources of losses are inherent to the use of plasmons during interference experiments and have different consequences when studying N00N states. First of all, there are linear losses of surface plasmon upon propagation over a distance $\mathrm{d}$. The question is therefore what are losses for a Fock state $|N\rangle$ with $\mathrm{N}$ particles. To get some insight into that question, we consider the phase variation $\varphi=k d$ of a single particle state due to propagation over a distance $\mathrm{d}$, with $k=\frac{2 \pi}{\lambda}$ the wavevector. As previously mentioned, this becomes $\varphi_{N 00 N}=k N d$ for the state $|N\rangle$. Introducing losses by considering that the wavevector $k=\frac{2 \pi}{\lambda}$ is complex $\mathrm{k}=\mathrm{k}^{\prime}+\mathrm{ik}$ ", we expect to observe a decay length of the state $\delta=\frac{1}{2 N k \prime^{\prime}}$ $\mathrm{N}$ times smaller than for a single particle state. We prove this result in [35]. The result can be interpreted with a naive picture: the transmission probability of each particle through $\mathrm{d}$ is given by $\exp (-d / \delta)$ so that the transmission probability of the $\mathrm{N}$ particles is given by $\exp (-N d / \delta)$. The second source of losses is due to the plasmonic beamsplitter itself. As experimentally shown the previous experiment, the presence of losses in the beamsplitter allows us tomodify the phase relation between the reflection and transmission factor. Depending on the phase relation of the input N00N state, one can deterministically obtain either a single photon state or a mixture of zero and two photon states at the output. This phenomenon has been called quantum nonlinear absorption. In this setup, evidence of being in such a coherent absorption regime is given by the reminiscent single plasmon oscillations in the inset of Fig. 4. Indeed, the 
observed phase shift between signals from SPCMs A and B is close to 0 it is the direct consequence of the phase relation $t= \pm r$. The in-phase evolution of the signals can be interpreted as the successive preferential transmission or absorption of single particles. We can therefore assume that the evolution of the N00N interference signal follows an analogous scheme: when the maxima are reached, the output state mix preferentially contains two-particle states ( thus increasing the number of coincidences). When the minima are reached, one gets more single particle states, thus reducing coincidence counts.

\section{CONCLUSION}

We have observed experimentally coalescence and anticoalescence of surface plasmons at a lossy beam splitter. The coalescence dip is obtained when orthogonality is achieved between $r$ and $t$, thus reproducing the results expected with a lossless beamsplitter. In that case, the dip in the coincidence proves the bosonic nature of SPPs. The anticoalescence peak can be obtained when $\mathrm{r}=+-\mathrm{t}$. This relation is allowed only in presence of $50 \%$ losses. When using a similar lossy beamsplitter to observe N00N state interferences, similar considerations lead to the observation of a quantum non-linear effect. In both situations, we experience quantum interference scenarios that affect lossy states, or in other workds, modify the output probabilities of two-particle states or one-particle states. It is thus possible to design components that are able to preferentially transmit only two-particle states or one-particle states.

\section{REFERENCES}

[1] R. H. Ritchie. Plasma losses by fast electrons in thin films. Phys. Rev.106, 874-881 (1957)

[2] A. V. Akimov et al., Generation of single optical plasmons in metallic nanowires coupled to quantum dots. Nature 450, 402-406 (2007).

[3] Y.-J. Cai, M. Li, X.-F. Ren, C.-L. Zou, X. Xiong, H.-L. Lei, B.-H. Liu, G.-P. Guo, G.-C. Guo. High-visibility on-chip quantum interference of single surface plasmons. Phys. Rev. Appl. 2, 014004 (2014).

[4] R. Kolesov et al., Wave-particle duality of single surface plasmon polaritons. Nat. Phys. 5, 470-474 (2009).

[5] M.-C. Dheur et al., Single-plasmon interferences. Sci. Adv.2, e1501574 (2016).

[6] E. Altewischer, M.P. Van Exter, J.P. Woerdman. Plasmon-assisted transmission of entangled photons. Nature 418, 304-306 (2002).

[7] S. Fasel et al.,Energy-time entanglement preservation in plasmon-assisted light transmission. Phys. Rev.Lett. 94, 110501 (2005).

[8] X. F. Ren, G. P. Guo, Y. F. Huang, C. F. Li, G. C. Guo. Plasmon-assisted transmission of high-dimensional orbital angular-momentum entangled state. EPL 76, 753 (2006).

[9] R.W. Heeres, L.P. Kouwenhoven, V. Zwiller. Quantum interference in plasmonic circuits. Nat. Nanotech. 8, 719-722 (2013).

[10] J.S. Fakonas, H. Lee, Y. A. Kelaita, H. A. Atwater. Two-plasmon quantum interference. Nat. Phot. 8, 317-320 (2014).

[11] S. Dutta Gupta, G. S. Agarwal. Two-photon quantum interference in plasmonics: theory and applications. Opt. Lett. 39, 390-393 (2014).

[12] G. Di Martino et al., Observation of Quantum interference in the Plasmonic Hong-Ou-Mandel Effect. Phys. Rev. Appl. 1, 034004 (2014).

[13] S. M. Wang et al., A $14 \times 14 \mu \mathrm{m} 2$ footprint polarization-encoded quantum controlled-not gate based on hybrid waveguide. Nat. Comm.7 (2016).

[14]E. Knill, R. Laflamme, G. J. Milburn. A scheme for efficient quantum computation with linear optics. Nature 409, 46-52 (2001).

[15] D. E. Chang, A. S. Sørensen, P. R. Hemmer, M. D. Lukin. Quantum optics with surface plasmons. Phys. Rev. Lett.97, 053002 (2006).

[16] R. Loudon. Fermion and boson beam-splitters statistics. Phys. Rev. A 58, 4904 (1998) 
[17] A. Zeilinger. Quantum Entanglement : A Fundamental Concept Finding its Applications. Phys. Script. 76, 203209 (1998).

[18] M. Michler, K. Mattle, H. Weinfurter, A. Zeilinger. Interferometric Bell-state analysis. Phys. Rev. A 53, R1209 (1996).

[19] J. C. F. Matthews et al., Observing fermionic statistics with photons in arbitrary processes. Sci. Rep. 3, 1539 (2013).

[20]P. G. Kwiat, A. M. Steinberg, R. Y. Chiao, Observation of a « quantum eraser » : A revival of coherence in a two-photon interference experiment. Phys. Rev. A. 45, 7729 (1992).

[21] S. M. Barnett, J. Jeffers, A. Gatti, R. Loudon. Quantum optics of lossy beam splitters. Phys. Rev. A 57, 2134 2145 (1998).

[22] J. Jeffers. Interference and the lossless lossy beam splitter. J. Mod. Opt.47, 1819-1824 (2000).

[23] T. Roger et al., Coherent perfect absorption in deeply subwavelength films in the single-photon regime. Nat.Comm. 6 (2015).

[24] T. Roger et al., Coherent Absorption of N00N states. Phys. Rev. Lett.117, 023601 (2016).

[25] C. Hong, Z. Ou, L. Mandel. Measurement of subpicosecond time intervals between two photons by interference. Phys. Rev. Lett. 59, 2044 (1987).

[26] A. Zeilinger, H. J. Bernstein, M. A. Horne. Information transfer with two-state two-particle quantum systems. $J$. Mod. Opt. 41 (12), 2375-2384 (1994).

[27] M. S. Tame et al., Single-photon excitation of surface plasmon polaritons. Phys. Rev. Lett. 101, 190504 (2008).

[28] A. Baron et al., Compact antenna for efficient and unidirectional launching and decoupling of surface plasmons. Nano Lett. 11, 4207-4212 (2011).

[29] M. U. González et al., Design, near-field characterization, and modeling of $45^{\circ}$ surface-plasmon Bragg mirrors. Phys. Rev. B73, 155416 (2006).

[30] E. Silberstein, P. Lalanne, J.-P. Hugonin, Q. Cao. Use of grating theories in integrated optics. JOSA A 18, 28652875 (2001).

[31] W Wan et al.,. Time-reversed lasing and interferometric control of absorption. Science 331, 889 (2011).

[32]Z. Ou, E. C. Gage, B. Magill, L. Mandel. Fourth order interference technique for determining the coherence time of a light beam. JOSA B 6, 100-103 (1989).

[33] Okamoto, R., Hofmann, H. F., Nagata, T., O'Brien, J. L., Sasaki, K., \& Takeuchi, S. (2008). Beating the standard quantum limit: phase super-sensitivity of N-photon interferometers. New Journal of Physics, 10(7), 073033.

[34] Vest, B., Dheur, M. C., Devaux, É., Baron, A., Rousseau, E., Hugonin, J. P., ... \& Marquier, F. (2017). Anticoalescence of bosons on a lossy beam splitter. Science, 356(6345), 1373-1376.

[35] Vest, B., Shlesinger, I., Dheur, M. C., Devaux, É., Greffet, J. J., Messin, G., \& Marquier, F. (2018). Plasmonic interferences of two-particle N00N states. New Journal of Physics, 20(5), 053050 